\title{
Immunotoxin-mediated targeting of claudin-4 inhibits the proliferation of cancer cells
}

\author{
S.M. HASHIMI, S. YU, N. ALQURASHI, D.S.IPE and M.Q. WEI \\ Division of Molecular and Gene Therapies, Griffith Health Institute and School of Medical Science, \\ Griffith University, Gold Coast, Queensland 4215, Australia
}

Received October 3, 2012; Accepted November 26, 2012

DOI: 10.3892/ijo.2013.1881

\begin{abstract}
Immunotoxins are engineered chimeric proteins that consist of a fragment of a toxin fused to a modified antibody or growth factor capable of targeting specific cells. Furthermore, these proteins can be targeted to receptors that are commonly overexpressed on cancer cells. The majority of immunotoxins function by binding to cells, translocating into the cytosol and inhibiting protein synthesis. In this study, the expression of claudin-4 (CLDN4) in various cancer cells was analysed as a potential target for immunotoxins. To target CLDN4expressing cancer cells, the c-terminal CLDN4-binding domain of Clostridium perfringens enterotoxin (CPE) was fused to the Pseudomonas aeruginosa exotoxin A (ETA) domain to create an immunotoxin (CPE-ETA'). Subsequently, the capacity of such an immunotoxin in suppressing the proliferation of CLDN4positive cancer cells was investigated. We report that head and neck squamous carcinoma cells (HN5) have an elevated CLDN4 expression compared to the other cell lines tested. Our findings further demonstrate that CPE-ETA' is highly potent against MCF-7 breast [50\% inhibitory concentration ( $\left.\left(\mathrm{IC}_{50}\right) 9.8 \mathrm{ng} / \mathrm{ml}\right]$ and $\mathrm{HN} 5$ head/neck $\left(\mathrm{IC}_{50} 8.8 \mathrm{ng} / \mathrm{ml}\right)$ cancer cell lines, while it has no cytotoxic effects on HeLa cells (CLDN4-negative). The immunotoxin was subsequently expressed in the tumour colonising oncolytic strain, Clostridium ghonii. Most importantly, the strictly anaerobic Clostridium ghonii was able to overexpress and secrete a functional CPE-ETA' fusion protein. Our findings open the possibility of the targeted delivery of the immunotoxin locally to tumour sites at a high concentration
\end{abstract}

Correspondence to: Professor Ming Q. Wei, Division of Molecular and Gene Therapies, School of Medical Science, Griffith University, Little High St., Gold Coast, Queensland 4215, Australia

E-mail: m.wei@griffith.edu.au

Abbreviations: CLDN4, claudin-4; ETA, Pseudomonas aeruginosa exotoxin A; CPE, Clostridium perfringens enterotoxin; EF-2, elongation factor-2; MTT, 3-(4,5-dimethylthiazol-2-yl)-2,5-diphenyltetrazolium bromide

Key words: claudin-4, head and neck cancer, immunotoxin, oncolytic Clostridium using strictly anaerobic Clostridium ghonii for the treatment of CLDN4-positive cancer cells.

\section{Introduction}

Cancer is a complex disease that requires the use of different methods and treatment modes to cure. Surgery, radiotherapy and chemotherapy, alone or in combination, are currently the prevalent treatment modalities (1). However, there is an urgent need for targeted therapies, which may provide more curative prospects.

Claudins (CLDNs) are a family of 17-27 kDa integral membrane tight junction (TJ) proteins that pass through the paracellular space in epithelial and endothelial tissues (2). The CLDN protein structure consists of a cytoplasmic N-termini, a C-termini and two extracellular loops. One of the loops interacts with CLDNs on adjacent cells to form an adhesive structure with other TJ proteins (3). The other extracellular loop in CLDN3 and CLDN4 serves as a binding site for Clostridium perfringens (C.perfringens) enterotoxin (CPE) (4).

As altered CLDN expression is frequently found in cancer cells, the hypothesis of a correlation between altered CLDN expression and cancer has been suggested (5). The loss of CLDNs and other TJ proteins in cancer has been interpreted as a mechanism for the loss of cell adhesion which is an important step in cancer progression and metastasis. However, many CLDNs, such as CLDN3 and CLDN4, are typically upregulated in many types of cancer, such as ovarian, breast, prostate, colon and pancreatic cancer, suggesting that they may have a positive effect on tumourigenesis and may lead to an increase in invasion, motility and cell survival (5). The potential value of CLDN in cancer therapy has been the subject of a number of studies (6) and is based on the fact that CLDNs are expressed at the cell surface and contain two extracellular domains that serve as potential target sites.

$\mathrm{CPE}$ is commonly associated with $C$. perfringens type A food poisoning. CPE is a single polypeptide of $35 \mathrm{kDa}$, which, upon binding to its receptors, causes cytolysis through its effects on membrane permeability (5,7). Both CLDN3 and CLDN4 are receptors for CPE. The intra-tumoural administration of CPE in cancer cells has been shown to result in tumour regression concomitant with a large degree of tumour necrosis (5). It was first demonstrated by Michl et al that the treatment of xenograft pancreatic cancer cells with CPE, led 
to a significant reduction in tumour burden, accompanied by necrosis, in CLDN4-expressing tumour cells (8).

Pseudomonas aeruginosa exotoxin A (ETA) functions by binding to nicotinamide and releasing the adenine dinucleotide (ADP-ribose) in mammalian cells. This ADP-ribose unit stays attached to ETA and is transferred to elongation factor-2 (EF-2), a protein involved in the translation and elongation of proteins. This, in turn, blocks protein synthesis in host cells, causing damage to target tissues (9). In this study, we demonstrate that HN5 head and neck squamous carcinoma cells overexpress CLDN4 and that targeting the CLDN4 receptor by CPE-ETA' (an immunotoxin created by fusing the c-terminal CLDN4-binding domain of CPE to the ETA domain) efficiently and specifically kills HN5 cells. Furthermore, our findings show that CPE-ETA' can be expressed and secreted by the oncolytic bacterial strain, Clostridium ghonii (C. ghonii), and that the secreted protein is potent against CLDN4-expressing cells.

\section{Materials and methods}

Cell culture conditions. The cell lines used in this study were the HN5 human head and neck squamous carcinoma, MCF-7 breast ductal carcinoma, A549 non-small cell lung cancer, MRC-5 normal foetal lung fibroblast, HT29 and HCT116 colon cancer, HeLa cervical cancer and Huh-7 hepatocarcinoma cells. The cancer cells were cultured in completed medium consisting of $500 \mathrm{ml}$ DMEM (Gibco), 10\% FBS, $12.5 \mathrm{ml}$ HEPES buffer solution $(1 \mathrm{M})$ and $1 \mathrm{ml}$ of penicillin $(5,000 \mathrm{U})$ and streptomycin $(5,000 \mu \mathrm{g})$ antibiotic mixture. Culture flasks were placed in a sterile tissue culture incubator under a humidified atmosphere at $37^{\circ} \mathrm{C}$ and $5 \% \mathrm{CO}_{2}$. Tissue culture was performed by routine procedures (10). All cell lines were purchased from the American Type Culture Collection, apart from HN5 which was kindly provided by Dr HongJian Zhu, University of Melbourne, Melbourne, Australia.

Confirmation of CLDN4 expression in cancer cell lines. Cells were seeded at appropriate densities and were cultured until $80-90 \%$ confluency. Subsequently, the cells were washed three times in PBS, scraped, centrifuged and resuspended in $100 \mu \mathrm{l}$ of cell extraction buffer (Invitrogen) with $1 \mathrm{mM}$ phenylmethanesulfonylfluoride (PMSF) and protease inhibitor on ice for $30 \mathrm{~min}$ while vortexing every $10 \mathrm{~min}$. The lysate was clarified by centrifugation at $13,000 \mathrm{rpm}$ at $4^{\circ} \mathrm{C}$ and the supernatant stored at $-80^{\circ} \mathrm{C}$ until further use. Total protein concentration was measured by spectrophotometry using the DC protein assay kit (Bio-Rad), and equal amounts of proteins were loaded onto SDS-PAGE gels for western blot analysis.

For qPCR analysis, RNA isolated from the cultured cells was converted to cDNA using SuperScript III (Invitrogen). Primers used for qPCR were as follows: CLDN4 forward, 5'-AGT GCA AGG TGT ACG ACT CGC T-3' and reverse, 5'-CGC TTT CAT CCT CCA GGC AGT T-3'. GAPDH and $\beta$-actin were used as the internal reference genes.

SDS-PAGE and western blot analysis. For SDS-PAGE, the following protein ladders were used: Precision Plus Protein Dual Color Standards (Bio-Rad), PageRuler Plus Prestained Protein Ladder (Fermentas). For western blot analysis, the primary antibodies used were: anti-His antibody, anti-CLDN4 antibody and anti- $\alpha$-tubulin antibody. The secondary antibody used was goat-anti-mouse IgG antibody. SDS-PAGE and western blot analysis were performed according to standard procedures (11).

Bacterial strains and plasmids. The properties of the bacteria and plasmids used in this study are listed in Table I.

Construction of Clostridial CPE-ETA' fusion vectors. To construct pMTL-10 ${ }^{\mathrm{His}}$-cCPE-ETA', p10 ${ }^{\mathrm{His}}$-cCPE-ETA' (12) was used as the template for PCR. The forward primer SfIICPE (5'-GAG GGC CCA GCC GGC CCA TCA TCA TCA TCA TCA TC-3') and the reverse primer SmaI_ETA (5'-GTC CCG GGA GTT ACT TCA GGT CCT CGC-3') were used to amplify $10^{\mathrm{His}}$-cCPE-ETA' which incorporated the SfiI and SmaI sites, respectively. PCR products were amplified using Phusion HighFidelity DNA Polymerase (Finnzymes) to minimise sequence errors. Finally, amplicons were excised from gels and cloned into the Clostridial shuttle vector, pMTL-555, using the restriction sites, SfiI and SmaI. The fusion protein is preceded by a secretion signal which is cleaved upon extracellular export of the protein. The recombinant plasmid pMTL-10 ${ }^{\text {His }}$-cCPE-ETA' was verified by sequencing.

Conjugal transfer of plasmids into $C$. ghonii. For conjugation Escherichia coli (E. coli) cultures were grown aerobically at $37^{\circ} \mathrm{C}$, while Clostridium cultures were grown anaerobically at $37^{\circ} \mathrm{C}$. After $25 \mu \mathrm{l}$ spots of $C$. ghonii overnight cultures were absorbed by HI agar (without antibiotic), CA434 donor cell suspensions (E. coli strain able to transfer the pMTL-555 vector to Clostridium via its helper plasmid capabilities) with recombinant vectors were spotted $(25 \mu \mathrm{l})$ on these Clostridium spots and grown at $37^{\circ} \mathrm{C}$, overnight in anaerobic conditions. To select Clostridia which had taken up the recombinant plasmid, the spots were spread on HI agar plates selected by $10 \mu \mathrm{g} / \mathrm{ml}$ erythromycin, $250 \mu \mathrm{g} / \mathrm{ml}$ cycloserine and $10 \mu \mathrm{g} / \mathrm{ml}$ polymyxin B. The plates were grown in anaerobic conditions until colonies were visible. To further verify that the Clostridium had taken up the plasmids, 15 colonies were selected from these plates and each colony was cultured under aerobic and anaerobic conditions, respectively. Since Clostridium can only grow under strict anaerobic conditions, there should only be growth under anaerobic conditions.

Preparation of Clostridial secreted proteins. Clostridia were cultured in $30 \mathrm{ml}$ of $\mathrm{HI}$ medium with erythromycin $10 \mu \mathrm{g} / \mathrm{ml}$ and D-cycloserine $250 \mu \mathrm{g} / \mathrm{ml}$ overnight at $37^{\circ} \mathrm{C}$ under anaerobic conditions. The cultures were spun at $5,000 \mathrm{x} g$ and the supernatant (medium) was filtered through a 0.2-micron Millex-HV Syringe-driven filter unit. The filtered medium was concentrated using an Amicon ultra centrifugal filter (ultracel-30k), at $5,000 \mathrm{rpm}$ for at least $30 \mathrm{~min}$ at $4^{\circ} \mathrm{C}$. The proteins were washed by PBS and concentrated 30 -fold to $500 \mu 1$. The DC protein assay kit (Bio-Rad) was used to measure protein concentrations.

Expression of CPE-ETA' in E.coli. To produce $10^{\mathrm{His}}$-cCPE-ETA', the $10^{\text {His }}$-cCPE-ETA' plasmid was used as previously reported by us (12). After transfection into E. coli, protein purification was performed using a Ni-NTA Fast Start kit (Qiagen) 
Table I. Bacterial strains and plasmids.

\begin{tabular}{|c|c|c|}
\hline Strain or plasmid & Relevant characteristics & Source \\
\hline \multicolumn{3}{|l|}{ Plasmids } \\
\hline p10 ${ }^{\text {His }}$-cCPE-ETA' & Amp, T7, N-His-tag, MCS & $(12)$ \\
\hline pMTL-555 & repL, traJ, ermB, fac 2 & $(33)$ \\
\hline pMTL-10 ${ }^{\text {His }}$-cCPE-ETA' & repL, traJ, ermB, fac 2 & This study \\
\hline \multicolumn{3}{|l|}{ Strains } \\
\hline \multicolumn{3}{|l|}{ E.coli } \\
\hline DH5a & Ф80dlacZAM15, $\Delta$ (lacZYA-argF)U169 & Promega \\
\hline CA434 & HB101 carrying the IncP $\beta$ conjugative plasmid, R702 & $(33)$ \\
\hline BL21 A1 & F-omp $\mathrm{T}$ hsdSB(rB-, mB-) gal dcm araB::T7RNAP-tetA & Invitrogen \\
\hline \multicolumn{3}{|l|}{ Clostridia } \\
\hline C. ghonii & & This study \\
\hline
\end{tabular}

following the manufacturer's instructions. Purified protein was desalted and concentrated in PBS using an ultrafiltration filter (Amicon Ultra-15-30 kDa cut-off). Finally, protein samples were stored at $-80^{\circ} \mathrm{C}$ in $20 \%$ glycerol PBS and when required protein concentrations were measured using the DC protein assay kit (Bio-Rad).

3-(4,5-Dimethylthiazol-2-yl)-2,5-diphenyltetrazolium bromide (MTT) cytotoxicity assay. Cancer cells were seeded at a density of $1 \times 10^{4}$ cells per well of a 96-well plate. After overnight incubation, recombinant proteins were added to the cells and incubated for $48 \mathrm{~h}$. To ascertain the specificity of CPE-ETA' for the CLDN4 receptor, HN5 cells were incubated with anti-CLDN4 antibodies (blocking of the CLDN4 receptor) for $1 \mathrm{~h}$ prior to the addition of CPE-ETA'. MTT assays were performed according to a standard procedure (13). The absorbency was measured by using a POLARstar Omega spectrophotometer from BMG Labtech. The results were converted to percentage proliferation compared to the control PBS group.

Statistical analysis. Statistical analysis was performed using GraphPad Prism 5 software. The significance level was 0.05 $(\mathrm{P}<0.05)$ and a Student's t-test was used to analyse the data. Experiments were performed three times and the data are presented as the means \pm standard error of the mean.

\section{Results}

Identification of high levels of CLDN4 expression in cancer cell lines. Cell lysates from cancer cell lines were separated by SDS-PAGE, transferred onto PVDF membranes and probed with anti-human CLDN4 and $\alpha$-tubulin antibodies. Subsequent analysis revealed that CLDN4 expression in the MCF-7, HN5, HT29 and HCT116 cancer cell lines was significantly upregulated (Fig. 1A). Furthermore, a weak expression was observed in the A549 cells, while CLDN4 expression was undetectable in the HeLa, MRC-5 and Huh-7 cell lines. Therefore, for all subsequent proliferation experiments, HeLa cells were used as the negative control. In addition, even protein loading was confirmed by the expression of the housekeeping protein,

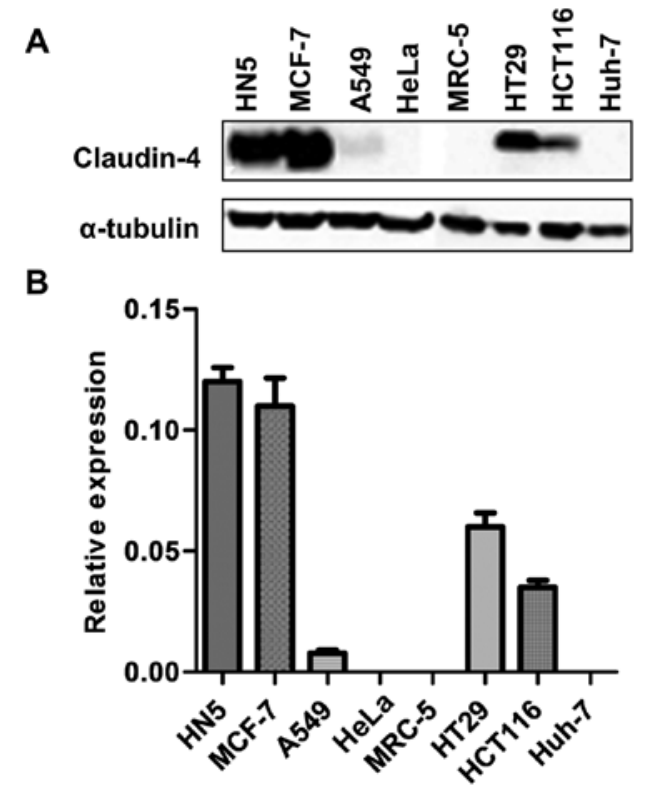

Figure 1. Detection of CLDN4 expression in cancer cells. (A) Proteins probed with anti-CLDN4 antibody followed by secondary antibody (goatanti-mouse). Equal protein loading of selected cancer cell lines (MCF-7, HN-5, A549, HeLa, MRC-5, HT-29, HCT116 and Huh-7) was confirmed by the housekeeping gene, $\alpha$-tubulin. (B) Real-time expression analysis of CLDN4 in cancer cell lines.

$\alpha$-tubulin. The results from real-time PCR analysis of the CLDN4 transcript were consistent with those obtained from western blot analysis (Fig. 1B).

CPE-ETA' protein expression and purification. His-tag purification was employed to purify CPE-ETA' (Fig. 2A) expressed in E. coli. Analysis of the purified protein by SDS-PAGE revealed a protein of the expected size $(58 \mathrm{kDa})$ in elution 1 and elution 2 (Fig. 2B). The purification step yielded $3 \mathrm{mg} / \mathrm{ml}$ of protein and a total of $6 \mathrm{mg}$ of protein was isolated.

Efficacy of purified CPE-ETA'. The toxicity of purified CPE-ETA' was examined by employing MTT proliferation assays (13). MTT is converted to formazan by living cells and can be detected by 
A

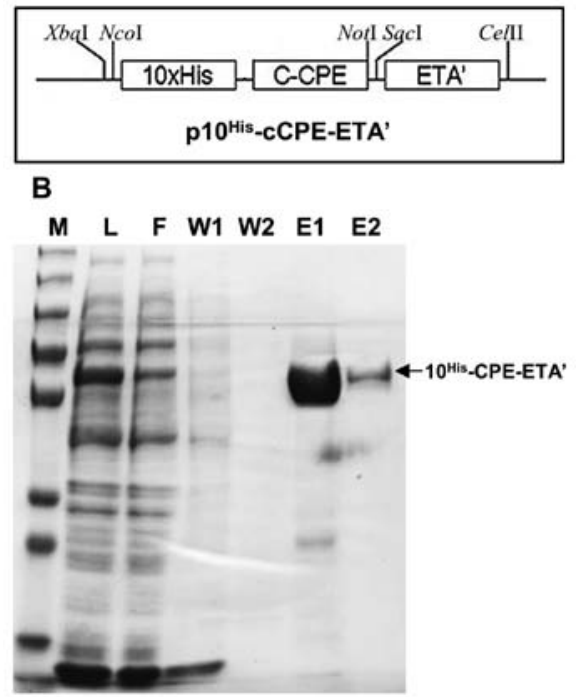

Figure 2. Construction and purification of $10^{\mathrm{His}}$-cCPE-ETA'. (A) $\mathrm{p} 10^{\mathrm{His}}$ cCPE-ETA' was constructed by Yao et al (12) in our laboratory. The backbone is pBM1.1 $\left(\mathrm{Kan}^{\mathrm{r}}\right)(21)$ and is induced by IPTG and L-arabinose for protein expression. (B) Protein purified by His-tag purification. Following electrophoresis, proteins were revealed by staining with Coomassie blue. M, page ruler plus prestained protein ladder; L, total cell lysate; F, column flowthrough; W1 and W2, wash one and two; E1 and E2, elution one and two.

spectrophotometric quantification. Purified protein was diluted from 0 to $40,000 \mathrm{ng} / \mathrm{ml}$ to give a dose response and subsequent calculation of the $50 \%$ inhibitory concentration $\left(\mathrm{IC}_{50}\right)$ values
(Fig. 3). PBS with $20 \%$ glycerol was used as the no-drug control. DMEM medium only (without cells and proteins) was used as the blank for MTT assay. The results showed that CPE-ETA' was very effective against HN5, MCF-7, HT29 and HCT116 cells with an $\mathrm{IC}_{50}$ between 8-160 ng/ml (Fig. 3). Furthermore, the A549 cells showed a moderate sensitivity against the targeted toxin $\left(\mathrm{IC}_{50} \sim 270 \mathrm{ng} / \mathrm{ml}\right)$, while the CLDN4-negative cell line, $\mathrm{HeLa}$, had an $\mathrm{IC}_{50}$ of $\sim 17,000 \mathrm{ng} / \mathrm{ml}$.

Specificity of CPE-ETA' for CLDN4 receptors. To examine the specific biniding of CPE-ETA' to the CLDN4 receptor, HN5 cells were incubated with an anti-CLDN4 antibody prior to the addition of CPE-ETA'. It was found that CPE-ETA' had no effect on cells pre-treated with the antibody compared to cells that were not treated with antibody (Fig. 4). It was also found that the CLDN4 antibody alone had no effect on the proliferation of the cells.

Construction of CPE-ETA'. Fig. 5 shows the map of CPE-ETA' constructed in the pMTL-555 backbone. pMTL-555 allows for the expression of proteins in Clostridium under the fac2 promoter. This plasmid is compatible in both $E$. coli and Clostridium. Furthermore, it contains elements for the conjugal transfer of plasmids from E. coli to Clostridium. $10^{\text {His }}$-cCPE-ETA' was amplified from $10^{\text {His }}$-cCPE-ETA' by PCR, Sfi and SmaI restriction enzyme sites (Fig. 5A) were incorporated into the amplicon for subsequent cloning into pMTL-555. The PCR fragment was cloned into pMTL-555 (digested by $S f$ I and $S m a \mathrm{I}$ ) to produce pMTL-10 ${ }^{\mathrm{His}}$-cCPE-
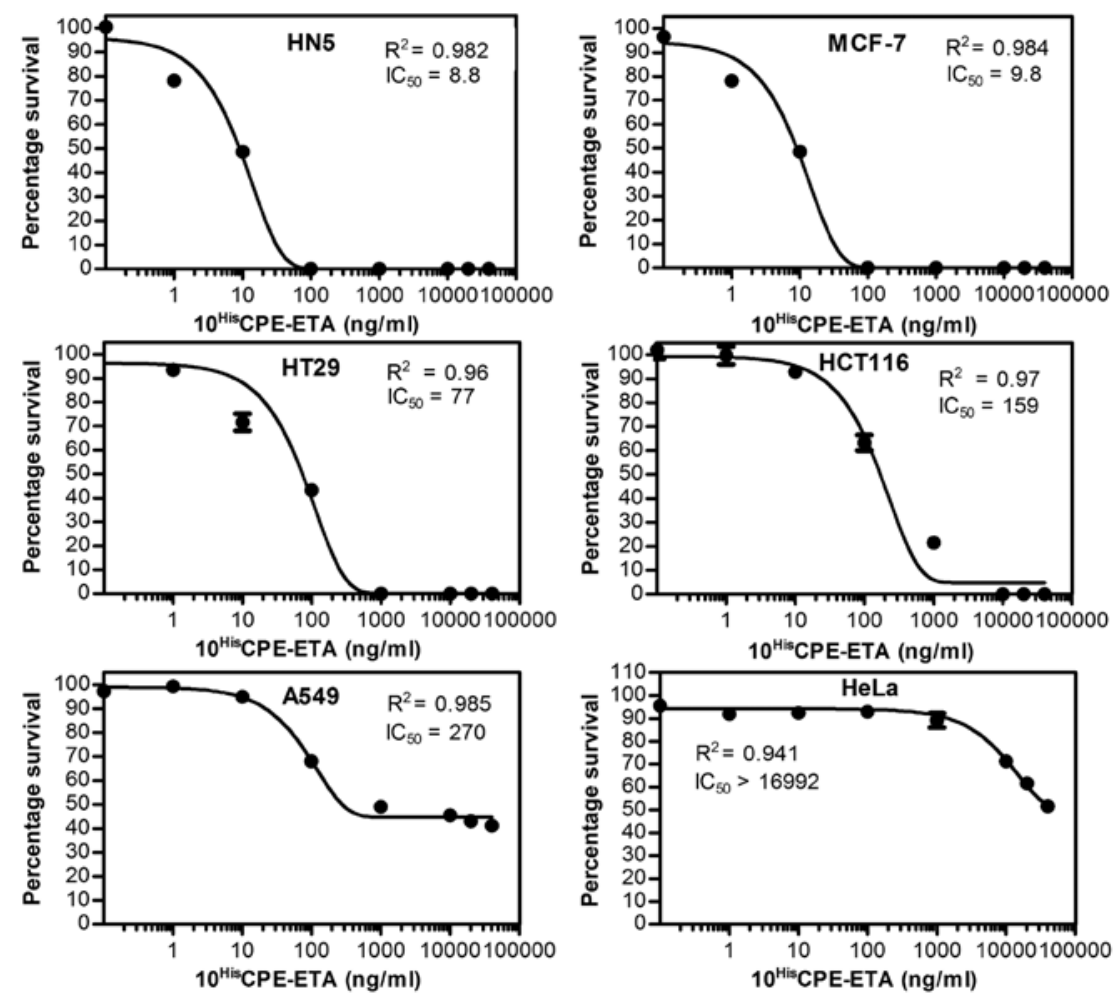

Figure 3. MTT assay was carried out on selected cancer lines. 10 ${ }^{\mathrm{His}}$-cCPE-ETA' protein toxicity tested in the cancer cell lines, A549, MCF-7, HN-5, HT-29, HTC116 and HeLa. Protein concentrations were from 0 to $40,000 \mathrm{ng} / \mathrm{ml}$. Each concentration of drug was repeated four times. Data represent the means \pm standard error. $10^{\mathrm{His}}$-cCPE-ETA' exhibited potent toxicity towards CLDN4-positive cells but had little effect on CLDN4-negative cells. HN5 and MCF-7 cells were the most sensitive $\left(\mathrm{IC}_{50} \sim 10 \mathrm{ng} / \mathrm{ml}\right.$ ), while HeLa cells were unaffected at concentrations $>10 \mu \mathrm{g} / \mathrm{ml}$. 
A

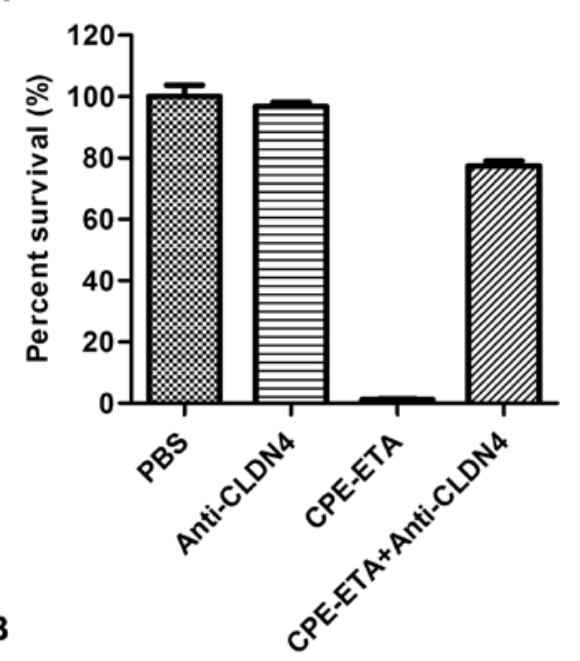

CPE-ETA

CPE-ETA + Anti-CLDN4

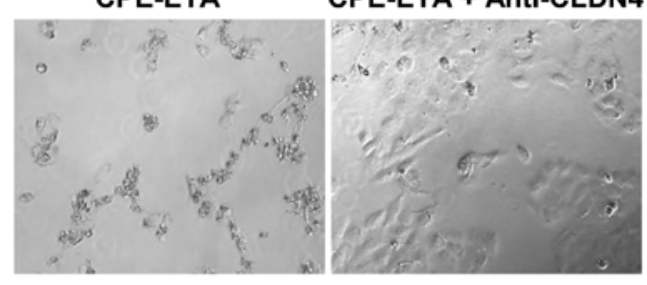

Figure 4. Anti-CLDN antibody abrogation of CPE-ETA' binding capacity (A) MTT assays were carried out to determine the binding specificity of CPE-ETA' for CLDN4. HN5 cells were seeded overnight in culture plates and treated as follows: PBS, no drug control; anti-CLDN4 (100 ng/ml); CPE-ETA' (100 ng/ml); anti-CLDN4 (100 ng/ml) plus CPE-ETA' $(100 \mathrm{ng} / \mathrm{ml})$. (B) Microscopic analysis of HN5 cells clearly show that pre-treatment with anti-CLDN4 antibody abrogates the effects of CPE-ETA'.

ETA' (Fig. 5A). Sequence analysis was used to verify the correct colonies in pMTL-555.

Transfer and expression of pMTL-10 ${ }^{\text {His }}$-cCPE-ETA' in C. ghonii. The E. coli donor strain, CA434, was used to transfer plasmids into Clostridium by conjugation. Clostridium is not easily amenable to heat- or electro-transformation of plasmid DNA (14). However, we demonstrate that conjugation can be used to transfer plasmid DNA into C. ghonii and, more importantly, this is the first report of the successful DNA transfer into the oncolytic $C$. ghonii strain. Transfer of the plasmid was achieved from CA434 by conjugation into $C$. ghonii after the selection of the plasmid by erythromycin and counter selection of E. coli by cycloserine and polymyxin. Clostridium is an obligate anaerobe and cannot grow in the presence of $\mathrm{O}_{2}$. Therefore, to confirm the identity of the recombinant Clostridium, 15 colonies were selected, plated on HI agar and grown under aerobic and anaerobic conditions, respectively. It was found that all colonies were able to grow under anaerobic conditions but were unable to grow under aerobic conditions (Fig. 5B), suggesting that all colonies were Clostridium.

For confirmation of recombinant protein expression, Clostridium strains were grown under anaerobic conditions overnight in HI medium. Protein from cell lysates secreted
A

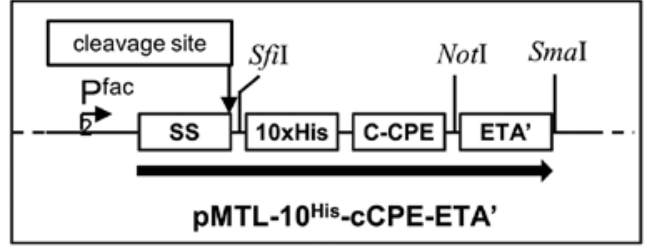

B

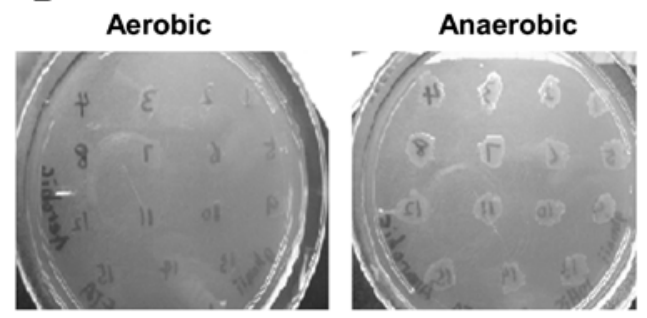

C

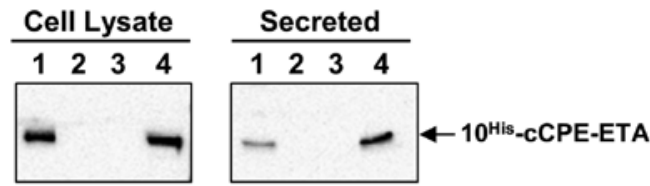

Figure 5. Construction of pMTL-10 $0^{\mathrm{His}}$-cCPE-ETA' and expression in Clostridium ghonii. (A) Indicated are restriction sites and important motifs highlighted are the promoter, the N-terminal secretion signal (SS), 10His, cCPE, and ETA'. Cloning of $10^{\mathrm{His}}$-cCPE-ETA' into pMTL-555 was achieved with $S f i$ and SmaI. Identification of transconjugants and detection of $10^{\mathrm{His}}$-cCPE-ETA' expression in Clostridium. (B) Bacterial cultures were replica-patched and grown under aerobic and anaerobic conditions at $37^{\circ} \mathrm{C}$ overnight. Growth was observed under anaerobic conditions only, indicating the presence of Clostridium. (C) Western blot analysis of $10^{\mathrm{His}}$-cCPE-ETA. The primary antibody used was anti-His antibody; the secondary antibody used was goat anti-mouse IgG antibody. Lanes 1 and 4, Clostridial colonies containing pMTL-10 ${ }^{\text {His }}$-cCPE-ETA; lanes 2 and 3, Clostridial colonies containing the empty vector, pMTL-555. Protein expression was detected in both the cell lysates and secretions in the medium in pMTL- $10^{\mathrm{His}}$-cCPE-ETA only.

into the growth medium was analysed by western blot analysis using an anti-His antibody. Subsequent western blot analysis showed that $10^{\mathrm{His}}$-cCPE-ETA' was expressed in Clostridium and was secreted into the medim (Fig. 5C).

Efficacy of CPE-ETA' secreted by $C$. ghonii. To examine the effects of CPE-ETA' secreted protein from $C$. ghonii, bacteria were grown in $\mathrm{HI}$ mediun overnight at $37^{\circ} \mathrm{C}$ under anaerobic conditions. The growth medium was concentrated using ultrafiltration and buffered in PBS. The proteins were used directly in MTT assays and the results expressed as the percentage survival. It was found that medium from non-recombinant Clostridium (pMTL-555) was able to kill all cells tested (Fig. 6), possibly due to endogenous toxins, protease and lipases produced by $C$. ghonii (15). Previous data from our group has shown that $C$. ghonii has oncolytic activity when administered in vivo in tumour-bearing mice with high specificity and safety profiles (unpublished data).

Furthermore, CPE-ETA' increased the killing capacity of C. ghonii-secreted protein in HN5, MCF-7 and HT29 cells (Fig. 6). It was found that CPE-ETA' did not affect the proliferation of CLDN4-negative HeLa cells. 

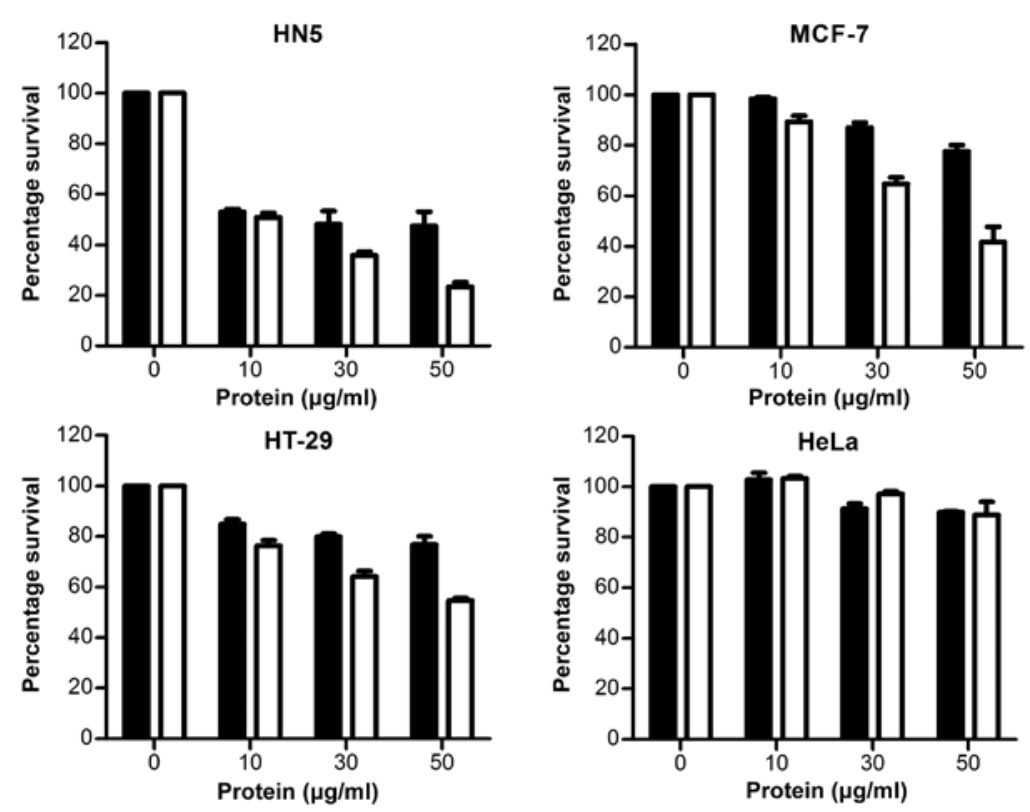

Figure 6. Cell proliferation assays of Clostridium-secreted proteins. Assays were performed in triplicate in 96-well plates. Black bars represent the empty vector control group (pMTL-555), while the pMTL-10 ${ }^{\mathrm{His}}$-cCPE-ETA group is represented by white bars. The cell lines tested were the HN5, HT29, MCF-7 cell lines, as well as HeLa cells (used as the negative control). Cells were seeded overnight and proteins were added. MTT assays were conducted $48 \mathrm{~h}$ after the addition of proteins. $10^{\text {His }}$-cCPE-ETA' was significantly different compared to the empty vector control at the highest protein concentration in HN5 (P=0.01), MCF-7 $(\mathrm{P}=0.0002)$ and HT29 cells $(\mathrm{P}=0.011)$, while the results for HeLa cells $(\mathrm{P}=0.07)$ were not significant. Data are presented as the means \pm standard error of the mean.

\section{Discussion}

The current study investigated the expression profiles of CLDN4 in a number of cancer cell lines. CLDN4 overexpression has been implicated in a variety of cancers, including breast, colon, prostate, pancreatic and ovarian cancers $(8,16-19)$. Furthermore, we investigated potential therapeutic strategies to target CLDN4. To this end, the binding domain of CPE (20), a natural toxin with high binding affinity for CLDN4, was employed to 'piggyback' the ETA toxin domain of Pseudomonas aeruginosa (21) to cancers overexpressing CLDN4 (12). The engineered immunotoxin upon binding to the CLDN4 receptor is expected to translocate into the cytoplasm via the Golgi apparatus and endoplasmic reticulum where it will bind to EF-2 and inhibit protein synthesis (Fig. 7). The fusion toxin was transfected ino E. coli and the purified protein was assayed against CLDN4-expressing cells. Finally, an oncolytic Clostridial (22) strain was engineered to secrete the CPE-ETA' fusion protein and the secreted protein was tested on several cancer cell lines.

Analysis of the expression levels of CLDN4 in the following cancer cell lines were found to be consistent with data from previous reports using these cell lines: MCF-7 human breast cancer (4), A549 human lung cancer (23), HeLa human cervical cancer (19), HT29 human colon cancer (24), HCT116 human colon cancer (25) and Huh-7 human liver cancer cells (26). On the other hand, the expression of CLDN4 in the HN5 human head and neck cancer and the MRC-5 human fibroblast cell line has not been previously reported.

Our findings show that purified CPE-ETA' was highly toxic to almost all the cancer cell types apart from the negative control cell line, HeLa. In addition, blocking the CLDN4 receptor with an anti-CLDN4 antibody abrogated the effects of CPE-ETA', suggesting that the killing capacity of CPE-ETA' is dependent on binding to the CLDN4 receptor (Fig. 7). The $\mathrm{IC}_{50}$ data indicated that this fusion protein had a significant effect on the HN5 human head and neck squamous carcinoma cancer cells, the MCF-7 human breast cancer and the HT29 human colon cancer cells. Furthermore, the high sensitivity of HN5 cells opens up the possibility of further research for the clinical application of CPE-ETA' as a therapeutic agent for the treatment of head and neck cancers.

Fifty percent of patients presenting with head and neck cancer are at an advanced stage of the disease, limiting effective treatment regimes (27). The standard therapy for head and neck cancer is chemo-radiotherapy which is often associated with serious side-effects (28). Targeted therapies have been developed which target the EGFR receptor, disrupting angiogenesis (an important process in the growth and progression of solid tumours) (29). Cetuximab, a monoclonal antibody, is clinically used for the targeted therapy of head and neck cancer (29). In this study, we show that CLDN4 is overexpressed in HN5 head and neck cancer cells and that CPE-ETA' is a potent inhibitor of HN5 cells by targeting the CLDN4 receptor. Furthermore, this killing capacity of CPE-ETA' was found to be specific and targeted to CLDN4-expressing cells, as shown by the inability of CPE-ETA' to inhibit the proliferation of CLDN4-negative HeLa cells (Fig. 3).

Having established the killing capacity of CPE-ETA' in HN5 cells, we sought to increase the specificity and availability of the CPE-ETA' in the tumour microenvironment. Clostridial strains have shown promise in seeking and colonising solid tumours. Since Clostridial species are obligate anaerobes, they can only replicate under hypoxic conditions, a hallmark of all solid tumours $(22,30)$. Furthermore, Clostridial species are spore-forming, which makes them ideal for carrying therapeutic payloads to solid tumours, as spores are known 


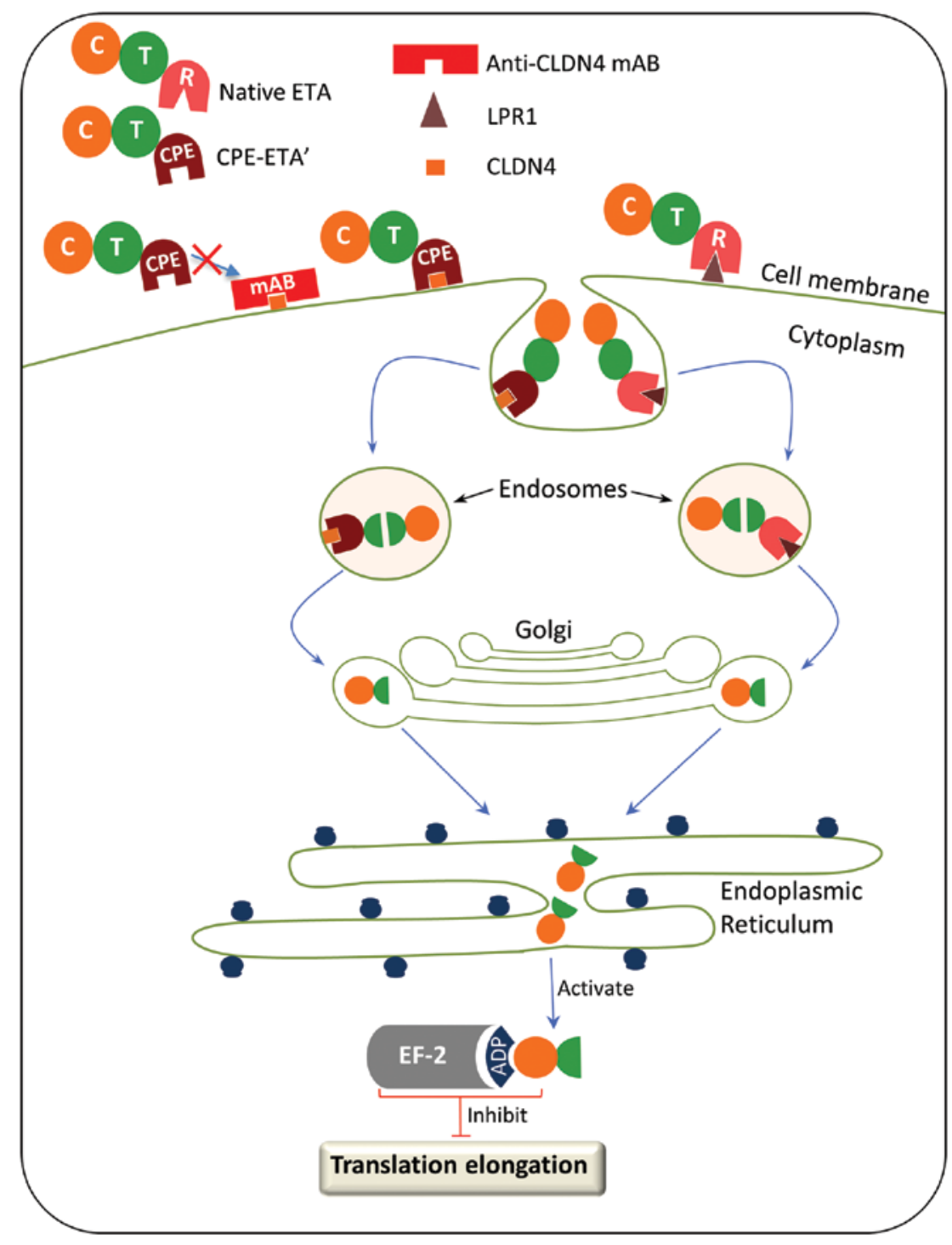

Figure 7. Mechanism of action of the CPE-ETA' immunotoxin. The native ETA protein binds the LPR1 receptor or in the case of CPE-ETA' it binds CLDN4. Upon binding the proteins undergo receptor-mediated endocytosis and subsequently within the endosome the $\mathrm{T}$ subunit is cleaved by furin proteases into two domains. The cleaved $\mathrm{C}+\mathrm{T}$ domain is translocated into the cytoplasm via the Golgi apparatus and endoplasmic reticulum. Finally, the $\mathrm{C}$ domain inactivates translation elongation factor-2 by enzymatic ADP-ribosylation. R, receptor-binding domain; T, translocation domain; C, (ADP)-ribosyltransferase A domain; LPR1, low-density lipoprotein receptor-related protein 1.

to elicit minimal immune response in the host (31). In order to examine the expression of functional CPE-ETA' fusion proteins in Clostridium, CPE-ETA' was used to construct the Clostridium expression vector, pMTL-555 (32). A Clostridium secretion signal was incorporated at the N-terminal of CPE-ETA' for extracellular secretion. The final construct was transferred to $C$. ghonii by conjugal transfer. Clostridium is not easily amenable to genetic modifications and is not readily transformed by heat shock or electroporation. This is the first report of successfully transforming $C$. ghonii and expressing functional therapeutic proteins.

Proteins secreted in the medium by recombinant $C$. ghonii were examined for cytotoxicity using MTT assay and were found to exert effects on several CLDN4-positive cancer lines. Furthermore, $C$. ghonii modified to express CPE-ETA' showed increased killing capacity in the HN5, HT29 and MCF-7 cells, but had little effect on HeLa cells (CLDN4-negative).
These data suggest that CPE-ETA' has high specificity for CLDN4-expressing cells. Therefore, in the tumour environment, the expression of this protein in $C$. ghonii will have little effect on CLDN4-negative cells. Furthermore, secretion of this protein from the tumour may be useful in eliminating distant small metastatic tumours that cannot be colonised by $C$. ghonii, as these tumours will not have the level of hypoxia to sustain the growth of $C$. ghonii.

In conclusion, this study confirms the upregulation of CLDN4 expression in certain cancer cell lines and identifies CLDN4 overexpression in the HN5 head and neck squamous carcinoma cell line. Furthermore, we demonstrate that the targeting of HN5 cells with the CLDN4 binding protein, CPE-ETA', not only shows extreme potency, but high specificity. In addition, we show that oncolytic Clostridia are capable of expressing and secreting the functional CPE-ETA' fusion protein and that this protein is capable of eliciting cell 
death in a number of CLDN4-positive cancer cells. The data presented in this study warrant further investigation for using oncolytic Clostridia to deliver therapeutic proteins locally to head and neck and breast cancer cells with high specificity, efficacy and safety.

\section{Acknowledgements}

This study was supported by the Dr Jian Zhou Smart State Fellowship from the Queensland state government and by grants from the National Health and Medical Research Council and Cancer Council of Queensland to M.Q.W. We would like to thank other members of the Wei Laboratory for their support and helpful comments.

\section{References}

1. Lucas R and Keisari Y: Innovative cancer treatments that augment radiotherapy or chemo-therapy by the use of immunotherapy or gene therapy. Recent Pat Anticancer Drug Discov 1: 201-208, 2006.

2. Krause G, Winkler L, Mueller SL, Haseloff RF, Piontek J and Blasig IE: Structure and function of claudins. Biochim Biophys Acta 1778: 631-645, 2008.

3. Lal-Nag M and Morin PJ: The claudins. Genome Biol 10: 235, 2009.

4. Saeki R, Kondoh M, Kakutani H, et al: A novel tumor-targeted therapy using a claudin-4-targeting molecule. Mol Pharmacol 76: 918-926, 2009

5. Morin PJ: Claudin proteins in human cancer: promising new targets for diagnosis and therapy. Cancer Res 65: 9603-9606, 2005 .

6. Suzuki M, Kato-Nakano M, Kawamoto S, et al: Therapeutic antitumor efficacy of monoclonal antibody against claudin-4 for pancreatic and ovarian cancers. Cancer Sci 100: 1623-1630, 2009.

7. Ling J, Liao H, Clark R, Wong MS and Lo DD: Structural constraints for the binding of short peptides to claudin-4 revealed by surface plasmon resonance. J Biol Chem 283: 30585-30595, 2008.

8. Michl P, Buchholz M, Rolke M, et al: Claudin-4: a new target for pancreatic cancer treatment using Clostridium perfringens enterotoxin. Gastroenterology 121: 678-684, 2001.

9. Pier GB, Boyer D, Preston M, et al: Human monoclonal antibodies to Pseudomonas aeruginosa alginate that protect against infection by both mucoid and nonmucoid strains. J Immunol 173: 5671-5678, 2004.

10. Phelan MC: Basic techniques in mammalian cell tissue culture. Curr Protoc Cell Biol Chapter 1: Unit 1 1, 2007.

11. Kurien BT and Scofield RH: Introduction to protein blotting. Methods Mol Biol 536: 9-22, 2009.

12. Yao Q, Cao S, Li C, et al: Turn a diarrhoea toxin into a receptormediated therapy for a plethora of CLDN-4-overexpressing cancers. Biochem Biophys Res Commun 398: 413-419, 2010.

13. Mosmann T: Rapid colorimetric assay for cellular growth and survival: application to proliferation and cytotoxicity assays. J Immunol Methods 65: 55-63, 1983.

14. Dong H, Zhang Y, Dai Z and Li Y: Engineering Clostridium strain to accept unmethylated DNA. PLoS One 5: e9038, 2010.

15. Barbé S, Van Mellaert L and Anné J: The use of clostridial spores for cancer treatment. J Appl Microbiol 101: 571-578, 2006.
16. Litkouhi B, Kwong J, Lo CM, et al: Claudin-4 overexpression in epithelial ovarian cancer is associated with hypomethylation and is a potential target for modulation of tight junction barrier function using a C-terminal fragment of Clostridium perfringens enterotoxin. Neoplasia 9: 304-314, 2007.

17. Lanigan F, McKiernan E, Brennan DJ, et al: Increased claudin-4 expression is associated with poor prognosis and high tumour grade in breast cancer. Int J Cancer 124: 2088-2097, 2009.

18. Ueda J, Semba S, Chiba H, et al: Heterogeneous expression of claudin-4 in human colorectal cancer: decreased claudin-4 expression at the invasive front correlates cancer invasion and metastasis. Pathobiology 74: 32-41, 2007.

19. Landers KA, Samaratunga H, Teng L, et al: Identification of claudin-4 as a marker highly overexpressed in both primary and metastatic prostate cancer. Br J Cancer 99: 491-501, 2008.

20. Kominsky SL, Vali M, Korz D, et al: Clostridium perfringens enterotoxin elicits rapid and specific cytolysis of breast carcinoma cells mediated through tight junction proteins claudin 3 and 4. Am J Pathol 164: 1627-1633, 2004.

21. Barth S, Huhn M, Matthey B, et al: Recombinant anti-CD25 immunotoxin RFT5(SCFV)-ETA' demonstrates successful elimination of disseminated human Hodgkin lymphoma in SCID mice. Int J Cancer 86: 718-724, 2000.

22. Wei MQ, Mengesha A, Good D and Anné J: Bacterial targeted tumour therapy-dawn of a new era. Cancer Lett 259: 16-27, 2008.

23. Frederick BA, Helfrich BA, Coldren CD, et al: Epithelial to mesenchymal transition predicts gefitinib resistance in cell lines of head and neck squamous cell carcinoma and non-small cell lung carcinoma. Mol Cancer Ther 6: 1683-1691, 2007.

24. Rangel LB, Agarwal R, D'Souza T, et al: Tight junction proteins claudin-3 and claudin- 4 are frequently overexpressed in ovarian cancer but not in ovarian cystadenomas. Clin Cancer Res 9: 2567-2575, 2003

25. Li J, Sherman-Baust CA, Tsai-Turton M, Bristow RE, Roden RB and Morin PJ: Claudin-containing exosomes in the peripheral circulation of women with ovarian cancer. BMC Cancer 9: 244, 2009.

26. Meertens L, Bertaux C, Cukierman L, et al: The tight junction proteins claudin- $1,-6$, and -9 are entry cofactors for hepatitis $C$ virus. J Virol 82: 3555-3560, 2008.

27. Gourin CG and Podolsky RH: Racial disparities in patients with head and neck squamous cell carcinoma. Laryngoscope 116: 1093-1106, 2006

28. Al-Sarraf M: Treatment of locally advanced head and neck cancer: historical and critical review. Cancer Control 9: 387-399, 2002.

29. Bonner JA, Harari PM, Giralt J, et al: Radiotherapy plus cetuximab for squamous-cell carcinoma of the head and neck. N Engl J Med 354: 567-578, 2006.

30. Wei MQ, Ipe D, Cao S and Hashimi S: Genetic Modification to Improve the Therapeutic Potential of Oncolytic Clostridia. In: Advances in Genetics Research. Urbano KV (ed). Vol 8. Nova Publishers, Hauppauge, NY, pp65-82, 2011.

31. Cao S, Cripps A and Wei MQ: New strategies for cancer gene therapy: progress and opportunities. Clin Exp Pharmacol Physiol 37: 108-114, 2010.

32. Theys J, Pennington O, Dubois L, et al: Repeated cycles of Clostridium-directed enzyme prodrug therapy result in sustained antitumour effects in vivo. Br J Cancer 95: 1212-1219, 2006.

33. Groot AJ, Mengesha A, van der Wall E, van Diest PJ, Theys J and Vooijs M: Functional antibodies produced by oncolytic clostridia. Biochem Biophys Res Commun 364: 985-989, 2007. 